\title{
Evolución de las creencias nucleares relacionadas con la adicción en drogodependientes con y sin trastornos de personalidad
}

\section{Changes in core beliefs associated with addiction among substance-dependent patients with vs. without comorbid personality disorders}

\author{
| José Miguel Martínez González*; \\ Antonio Verdejo García **
}

*Centro Provincial de Drogodependencias de Granada.
${ }^{*}$ Universidad de Granada.

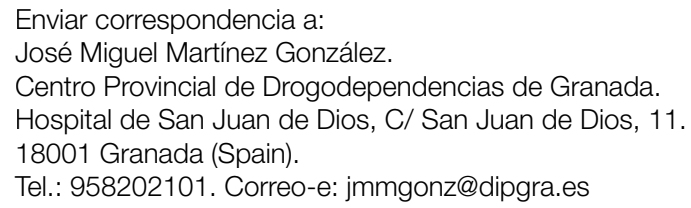

Tel.: 958202101. Correo-e: jmmgonz@dipgra.es

\section{Resumen}

Los objetivos de este estudio fueron: 1) Estimar la capacidad predictiva de las creencias sobre el craving y los consumos de alcohol; y 2) Examinar la evolución a lo largo de nueve meses de las creencias nucleares relacionadas con la adicción en función de la comorbilidad con trastornos de personalidad. Participaron 65 pacientes con dependencia de alcohol de los que el $56.4 \%$ presentaban TP comórbidos. Se administró el Cuestionario de Creencias relacionadas con la adicción y con el craving, y se recogieron como medidas de resultado el número de consumos de alcohol durante el tratamiento y el grado de ajuste psicológico. La relación entre los ítems del cuestionario y las variables de resultado se exploraron mediante regresiones lineales y la evolución de las creencias a lo largo del tratamiento se analizó con pruebas t para muestras relacionadas. Los resultados indican que es posible medir de manera fiable un conjunto de creencias adictivas, que el nivel de identificación con estas creencias permite predecir el craving, los consumos de alcohol durante el tratamiento y el ajuste psicológico global, y que el curso de dichas creencias durante el tratamiento difiere en función de la presencia de comorbilidad con TP; los pacientes con TP comórbido presentan una evolución mucho más irregular de su identificación con las creencias relacionadas con la adicción.

Palabras clave: longitudinal, creencias, trastornos de la personalidad, alcohol, tratamiento.

\section{Abstract}

The aims of this study were: 1) To evaluate the predictive ability of cognitive beliefs about craving and alcohol use during treatment; and 2) To examine the course of cognitive beliefs, over a nine-month follow-up, as a function of comorbidity with personality disorders (PDs). Participants in the study were sixty-five alcohol-dependent patients initiating treatment at the Provincial Drug-Dependence Centre in Granada (Spain), 56.4\% of whom had comorbid personality disorder. We administered the "Questionnaire on beliefs related to addiction and craving", taking number of alcohol-use episodes during treatment and degree of psychological adjustment as outcome variables. We used linear regression models to analyze the relation between questionnaire items and outcome variables, and related-samples t-tests to examine the course of beliefs throughout the follow-up. Results indicate that it is feasible to reliably measure a range of dysfunctional beliefs associated with addiction, that the degree of identification with these beliefs significantly predicts craving and alcohol use during treatment, and that the course of identification with these beliefs is considerably more irregular in alcohol-dependent individuals with comorbid PDs.

Key words: longitudinal, beliefs, personality disorders, alcohol, treatment. 
E modelo cognitivo de las drogodependencias postula la existencia de un estrecho vínculo entre las creencias disfuncionales relacionadas con la adicción (p.e., "creo que nunca podré superar mi adicción") y las variables mediadoras y de resultado del éxito del tratamiento, como el craving, las expectativas de autoeficacia y las recaídas (Beck, 1995). Por tanto, el modelo propone que la modificación de las creencias nucleares relacionadas con la adicción favorecerá sustancialmente el proceso de recuperación de los pacientes drogodependientes. Por este motivo, una parte fundamental de la terapia cognitiva de las adicciones consiste en identificar las creencias que influyen significativamente en el craving y el curso del tratamiento (Iraurgi y Corcuera, 2008; Merikle, 1999), siendo uno de los objetivos centrales de esta terapia hacer consciente al paciente sobre la conexión entre estas creencias, el deseo y las conductas de consumo (Beck, Wright, Newman y Liese, 1999).

Los contenidos de las creencias nucleares relacionadas con la adicción en pacientes drogodependientes hacen referencia tanto a la funcionalidad del consumo -pensamientos permisivos hacia el mismo - (Nuewman y Ratto, 1999), como a otras atribuciones sobre la propia capacidad de recuperación o sobre la utilidad del tratamiento y del soporte recibido. Las bajas expectativas de autoeficacia y de resultados tienen gran impacto sobre la aparición del craving y, consecuentemente, sobre la efectividad del tratamiento (Chicharro, Pedrero y Pérez, 2007; DiClemente, Fairhurst y Piotrowski, 1995). Asimismo, el hecho de que los pacientes perciban el tratamiento como útil incrementa significativamente la eficacia del mismo (Long, Williams y Hollin, 1999; Tiffany, 1990).

En un estudio reciente pudimos constatar la vinculación entre las creencias y el deseo de consumo de drogas, o craving (Martínez-González y Verdejo-Garcia, 2011). Mediante el uso de modelos de regresión jerárquica, demostramos que los niveles de identificación de los pacientes con las creencias nucleares relacionadas con la adicción -en particular con las vinculadas a la funcionalidad del consumo y las expectativas negativas de autoeficacia- constituian el mejor predictor de la intensidad del craving. Los resultados también mostraron que los niveles de craving se atenúan conforme incrementa la duración del tratamiento, presumiblemente debido a la eliminación progresiva de estas creencias. En contraste, la persistencia de estas creencias disfuncionales podría contribuir a explicar el hecho de que algunos pacientes puedan seguir presentando altos niveles de craving tras un periodo de abstinencia en el que cabe esperar la inapetencia de drogas. La evolución de los niveles de identificación del paciente con las creencias nucleares relacionadas con la adicción es especialmente importante durante los tres primeros meses de tratamiento, ya que a partir de ese momento el nivel de deseo de consumo no puede atribuirse al tiempo de abstinencia (López y Becoña, 2006; Martínez-González y Verdejo-García, 2011).

El tratamiento cognitivo de las adicciones persigue la progresiva modificación de las creencias nucleares relacionadas con la adicción como palanca de cambio de la conducta de consumo y como garante de la abstinencia. No obstante, uno de los factores centrales que condicionan la aplicación de esta modalidad de tratamiento y el curso de recuperación de la adicción es la comorbilidad de ésta con los trastornos de personalidad (TP) (Pennay, Cameron, Reichert, Strickland, Lee, Hall y Lubman, 2011). En estos pacientes con patología dual asociada al Eje II aparecen más dificultades para modificar las creencias dado que tienden a ser más estables $y$, por tanto, presentan más resistencia a ser modificadas (Beck, 2007). En consecuencia, es necesario analizar de manera diferencial la evolución de las creencias nucleares relacionadas con la adicción a lo largo del tratamiento en función de la coexistencia de TP.

En este trabajo, que constituye un estudio parcial de una investigación más amplia (ver Martínez-González, Graña y Trujillo, 2011), pretende mostrar el impacto de las creencias en el deseo de consumo y conocer la evolución de las mismas a lo largo del tratamiento dependiendo de la presencia de TP. Nos planteamos como objetivos específicos: 1) Estimar la capacidad predictiva de las creencias evaluadas sobre variables mediadoras y de resultado del tratamiento, incluyendo: i) las creencias relativas al craving, ii) los consumos de alcohol durante los tres primeros meses, y iii) el nivel de ajuste psicológico global. 2) Examinar la evolución de las creencias nucleares relacionadas con la adicción a lo largo del tratamiento, analizando las diferencias que pudieran derivarse de la presencia de TP comórbidos. Hipotetizamos que en la medida en que los pacientes se idenifiquen más con las creencias aumentará significativamente el nivel de craving y el número de consumos de alcohol, a la vez que disminuirá el nivel de ajuste psicológico global. Estas creencias disfuncionales se reducirán a partir de los 3 meses de tratamiento, aunque serán más resistentes en los pacientes con TP comórbidos.

\section{Método}

\section{Participantes}

La muestra, que ha sido objeto de otros trabajos (Martínez-González, Graña y Trujillo, 2010, 2011), está formada por 65 pacientes que cumplian criterios para la dependencia del alcohol (DSM-IV-TR, 2002) e iniciaban tratamiento en el Centro Provincial de Drogodependencias de Granada. Los participantes se seleccionaron de acuerdo con los siguientes criterios de inclusión: (a) debian ser personas que no habian estado en tratamiento, al menos en los dos meses previos al inicio del estudio; (b) debian estar abstinentes durante al menos dos semanas para poder llevar a cabo el diagnóstico de posibles trastornos comórbidos; y (c) debían encontrarse en el primer mes de tratamiento para homogeneizar los efectos prospectivos de la intervención terapéutica. En relación con las características demográficas de la muestra, el 70.8\% $(n=46)$ de los participantes son hombres, mientras que el $29.2 \%(n=19)$ son mujeres; la edad media es de 43.26 años. En cuanto a sus características clínicas, los participantes presentan una media de 11.83 años de evolución de la adicción y en el momento de la evaluación contaban con una media de 29 días de abstinencia. El $67.2 \%(n=44)$ de la muestra fue diagnosticado con patología dual: el $23.6 \%(n=15)$ en el Eje I y el $56.4 \%(n=$ 36) en el Eje II. 


\section{Instrumentos de evaluación}

El diagnóstico de Eje I se llevó a cabo mediante entrevista clínica, atendiendo a criterios DSM (DSM-IV-TR, 2002). EI diagnóstico de los TP del Eje II se realizó mediante la entrevista semiestructurada "Examen Internacional de los Trastornos de la Personalidad (IPDE)" (Loranger, 1995; versión española de López-lbor, Pérez Urdániz, y Rubio, 1996).

Para abordar el objetivo principal del estudio, la evaluación de las creencias nucleares relacionadas con la adicción y su evolución a lo largo del tratamiento, se administró un cuestionario de 23 ítems diseñados para medir el grado de identificación del paciente con una serie de creencias relacionadas con el craving, la funcionalidad del consumo, las expectativas de autoeficacia y de resultados del tratamiento, adherencia al tratamiento, mantenimiento de la abstinencia a largo plazo, creencias básicas adictivas y percepción de necesidad de ayuda de profesionales (Anexo 1). Este cuestionario utiliza una escala de respuesta tipo Likert, con cinco valores, que van desde "ningún grado de identificación con las creencias" a "muchísimo".

Como medidas de resultado se utilizaron: (i) el número de consumos de alcohol ocurridos durante los tres primeros meses de tratamiento (información obtenida fundamentalmente a partir de la información de los familiares y el propio paciente y confirmada con hemograma a los tres meses de tratamiento), y (ii) el ajuste psicológico global de los pacientes, medido con el Cuestionario General sobre Salud (General Health Questionnaire-GHO-28; Goldber y Hiller, 1979). Este cuestionario permite obtener un índice de gravedad del ajuste psicológico construido a partir de la suma de cuatro subescalas: sintomas somáticos, ansiedad, disfunción social y depresión (González-Saiz, Carulla, Martínez, López, Ruiz y Guerra, 1997). En este estudio, la interpretación del GHQ-28 se ajustó a un modelo dimensional en el que la puntuación total se considera dentro de un continuo hipotético bienestar-malestar psicológico; puntuaciones inferiores representan mejor estado de salud psicológico.

\section{Procedimiento}

Llevamos a cabo cuatro evaluaciones a lo largo del tratamiento. La primera evaluación se realizó durante el primer mes de tratamiento, en las condiciones que se han descrito anteriormente. La segunda evaluación se realizó a los tres meses del comienzo del tratamiento, la tercera a los seis y la cuarta a los nueve. En las tres evaluaciones de seguimiento se estableció un intervalo temporal de una semana para completar los distintos instrumentos. Aquellos pacientes que, por alguna razón, no completaban los cuestionarios en la semana designada quedaban excluidos del estudio. Estas condiciones restrictivas nos permitieron homogeneizar el tiempo de evolución en las evaluaciones de seguimiento, pero también generó, junto a los abandonos de tratamiento, un considerable nivel de muerte experimental: en la primera evaluación participaron 65 pacientes, en la segunda 31,21 en la tercera y 17 en la cuarta.

\section{Diseño y análisis estadístico}

Se empleó un diseño longitudinal, en el contexto de una metodología cuasi-experimental. La capacidad predictiva de las creencias relacionadas con la adicción y el proceso de tratamiento sobre el craving, los consumos de alcohol durante los primeros tres meses y el ajuste psicológico global se examinaron mediante análisis de regresión lineal. En estos análisis se incluyeron dos tipos de predictores de interés: i) la puntuación media del cuestionario de creencias, y ii) las puntuaciones de los items que consideramos fundamentales desde un punto de vista clínico.

El cuestionario, además de los ítems relativos al craving, incorpora otros relacionados con dimensiones clínicas de relevancia en el proceso de tratamiento: las expectativas de autoeficacia, las expectativas de resultado, el malestar emocional, y la falta de renuncia al consumo. Por tanto, siguiendo la misma metodologia con la que se analizaron las creencias referidas al craving, con un alto nivel de consistencia interna (ver Martínez-González, Graña y Trujillo, 2011), estudiamos la consistencia interna de estas otras cuatro dimensiones, agrupando los ítems que conforman cada dimensión según criterios de coherencia y relevancia clínica.

A continuación, los análisis de regresión incorporaron como predictores la puntuación media del cuestionario y los ítems más representativos de cada una de estas dimensiones, motivado por el interés clínico del estudio pormenorizado del contenido de determinadas creencias.

Las diferencias en el grado de identificación con las creencias en función de la comorbilidad con TP se analizaron mediante pruebas $t$ para muestras independientes y su evolución a lo largo del tratamiento se exploró mediante gráficos descriptivos y pruebas $t$ para muestras relacionadas.

Todos los análisis estadísticos se llevaron a cabo en el paquete estadístico PASW v. 18.

\section{Resultados}

El cuestionario de creencias relacionadas con la adicción y su tratamiento (CCAT) arrojó un $\alpha$ de Cronbach de .83 para el conjunto de los items, reflejando un nivel de consistencia interna adecuado. Esto nos permite utilizar la puntuación media del cuestionario como un indice del grado en que los pacientes se identifican con creencias vinculadas con la adicción y su tratamiento.

Adicionalmente, los análisis sobre las dimensiones especificas que conforman el cuestionario mostraron buenos índices de consistencia interna: expectativas negativas de resultados (items 4,12 y $14, \alpha$ de Cronbach de .75), expectativas positivas de autoeficacia (ítems $5,11,17$ y $8, \alpha=.65$ ), malestar emocional (items 6 y $7, \alpha=.56$ ), y falta de renuncia al consumo (items 1,9 y $15, \alpha=.71$ ). La dimensión de craving mostró ya en un estudio previo un $\alpha=.89$. Otras creencias están vinculadas con la ayuda familiar o la motivación al tratamiento.

\section{Capacidad predictiva de las creencias sobre el cra- ving, los consumos de alcohol y el ajuste global}

Creencias con capacidad de predecir el craving. El análisis de la capacidad predictiva de la creencia específica "creo que 
tengo la capacidad necesaria para superar mi drogodependencia" mostró que de manera significativa, cuanto mayores son las expectativas de autoeficacia menores son los niveles de craving (Beta $\left.=-.354 ; p=.004 ; \mathrm{R}^{2}=.125\right)$. De manera complementaria, la creencia "creo que nunca podré superar mi drogodependencia" (Beta $\left.=.354 ; \mathrm{p}=.004 ; \mathrm{R}^{2}=.146\right)$ predice positivamente los niveles de craving.

A continuación analizamos la correlación entre la media de los ítems vinculados con la "renuncia al consumo" y las creencias sobre el craving. Los resultados mostraron la existencia de una correlación positiva significativa entre ambos grupos de ítems ( $r=.448 ; p=.000)$, indicando que un bajo nivel de "renuncia" al consumo incrementa el deseo de consumo. Los modelos de regresión incluyendo las creencias vinculadas con el concepto de falta de renuncia mostraron resultados dispares para los distintos items que se refieren a este concepto. Mientras que la creencia "algún día consumiré aunque solo sea una vez" predice significativamente los niveles de craving (Beta =.303; $p=.017 ; R^{2}=.092$ ), las creencias "creo que podré tomar [alcohol] un día y no seguir haciéndolo" y "algún día consumiré de forma controlada" no predicen el deseo de consumo.

El análisis más detallado de la relación entre el deseo de consumo evaluado a través del ítem "ahora tengo ganas de consumir" y la creencia "algún día volveré a consumir aunque solo sea una vez" mostró que la relación entre estas dos creencias es positiva y estadísticamente significativa en las cuatro evaluaciones: inicio de tratamiento $(r=.286 ; p=.015)$, tres meses ( $r=$ $.487 ; p=.012)$, seis meses $(r=.640 ; p=.001)$, y nueve meses $(r$ $=.568 ; p=.009$ ). Esto es, a lo largo del tratamiento se constata que la creencia "algún día consumiré aunque solo sea una vez" se relaciona con un mayor nivel de deseo de consumo.

Creencias que predicen el consumo de alcohol a lo largo de los tres primeros meses de tratamiento. El $60 \%$ de los pacientes presentaron algún consumo de alcohol a lo largo de los tres primeros meses de tratamiento, no existiendo diferencias estadísticamente significativas en relación al número de consumo en función de la presencia de TP. La correlación entre la puntuación media del cuestionario de creencias y los consumos de alcohol no mostró valores estadísticamente significativos.

El análisis de la capacidad predictiva de la creencia especifica "creo que tengo la capacidad necesaria para superar mi drogodependencia" mostró que ésta se relaciona negativamente con el número de consumos (Beta= -.328; $p=.036 ; R^{2}=.108$ ). Es importante destacar que, mientras que la creencia "creo que tengo la capacidad necesaria para superar mi drogodependencia" predice el número de consumos, la creencia "creo que voya superar mi drogodependencia" no predice estos consumos.

El nivel de craving experimentado al iniciar el tratamiento predice el número de consumos a lo largo de los tres primeros meses (Beta $=.343 ; p=.028 ; R^{2}=.118$ ), de modo que un mayor nivel de deseo incrementa la posibilidad de consumir en las primeras etapas del tratamiento.

Asimismo, mostraron una capacidad predictiva significativa sobre el número de consumos que se producen a lo largo de los tres primeros meses de tratamiento las creencias sobre el craving "cuando se me mete en la cabeza consumir no puedo evitar hacerlo" (Beta $\left.=.312 ; p=.050 ; \mathrm{R}^{2}=.098\right)$, "tengo ganas de consumir cuando me siento mal" (Beta = .385; $p=.013 ; \mathrm{R}^{2}$ $=.148$ ), "tengo ganas de consumir cuando dispongo de dinero" (Beta $\left.=.360 ; p=.010 ; \mathrm{R}^{2}=.130\right)$ y "ahora tengo ganas de consumir" (Beta $\left.=.400 ; p=.010 ; R^{2}=.160\right)$. En todos los casos el sentido de la predicción es positivo -indicando que un mayor grado de identificación con estas creencias incrementa la probabilidad de consumir durante los tres primeros meses de tratamiento.

Creencias y ajuste psicológico global -GHQ-28. La puntuación media de las creencias que componen el cuestionario predice significativamente el índice global de gravedad de ajuste psicológico (Beta=.472; $p=.000 ; \mathrm{R}^{2}=.222$ ). Además predice las puntuaciones específicas de las escalas de ansiedad (Beta $\left.=.448 ; p=0.001 ; R^{2}=.201\right)$, depresión (Beta $=.347$; $\left.p=.009 ; R^{2}=.121\right)$, sintomas somáticos $\left(\right.$ Beta $=.415 ; p=.001 ; R^{2}$ $=.172)$, y relaciones sociales $\left(\right.$ Beta $\left.=.379 ; p=.004 ; R^{2}=.143\right)$.

Por otro lado, se analizó la capacidad predictiva del índice de gravedad del ajuste psicológico global como variable influyente en las creencias. Se constató que esta variable predice el grado con el que los pacientes se identifican con las creencias. Particularmente, destaca que la identificación con la creencia "algún dia consumiré aunque solo sea una vez" está determinado por la gravedad del ajuste psicológico (Beta $=.288$; $p=.033$; $\mathrm{R}^{2}=.083$ ).

También se constata que el índice de gravedad de ajuste psicológico predice el nivel con el que los pacientes se identifican con el conjunto de creencias que denominamos falta de renuncia al consumo (Beta $\left.=.264 ; p=.049 ; R^{2}=.070\right)$ de lo que se desprende que un mal ajuste psicológico dificulta la renuncia definitiva al consumo (Figura 1).

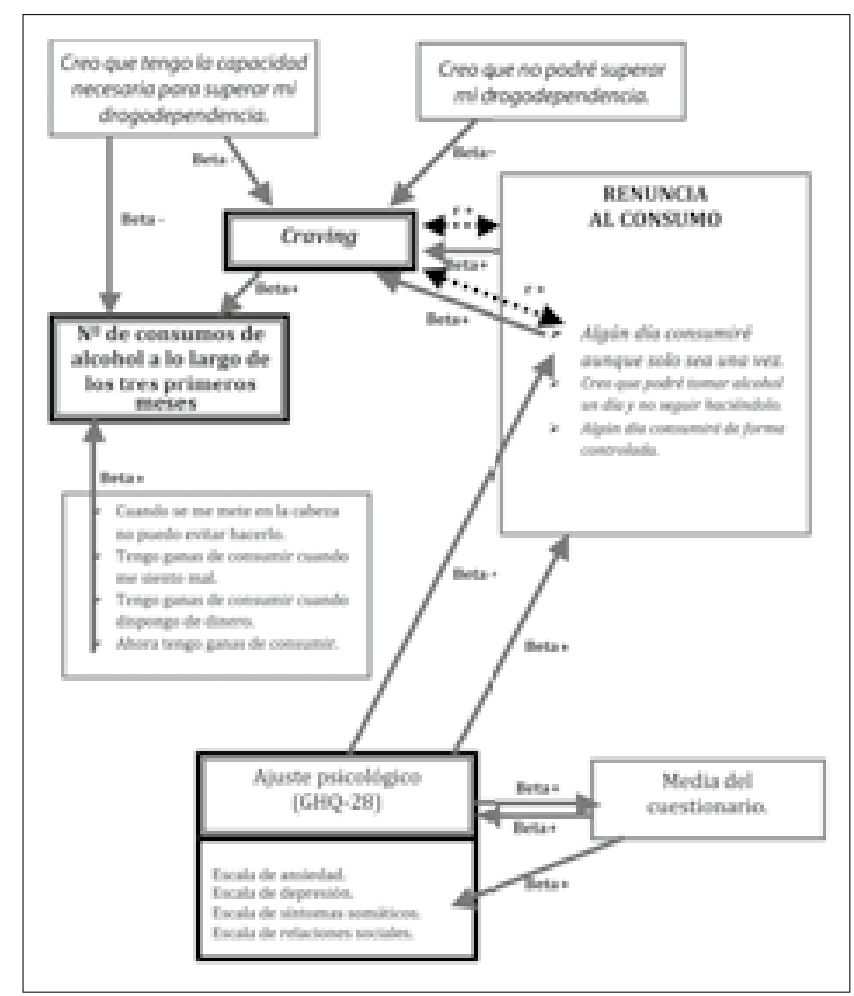

Figura. 1. Signo de Beta en las predicciones y correlaciones significativas. 


\section{Creencias y comorbilidad con Trastornos de Perso- nalidad (TP).}

Diferencias en función de la comorbilidad con TP. No se observaron diferencias estadísticamente significativas en la puntuación media del cuestionario al iniciar el tratamiento en función de la presencia o ausencia de comorbilidad con TP.

En relación a la creencia "creo que tengo la capacidad para superar mi drogodependencia" tampoco se observan diferencias estadísticamente significativas en función de la presencia de TP. No obstante, los resultados muestran que los pacientes que no presentan TP cuentan con mejores expectativas de autoeficacia en la primera evaluación (media no TP $=3.56$; $\mathrm{TP}=3.33$ ), segunda (media no $\mathrm{TP}=2.89 ; \mathrm{TP}=2.11$ ) y cuarta (media no TP $=2.89 ; \mathrm{TP}=2.11$ ).

Los análisis del item "tengo problemas emocionales con independencia de mi adicción", mostraron que los pacientes con TP son más conscientes de presentar estos problemas ( $M$ $-\mathrm{TP}=2.44 ; M-$ no TP= $1.56 ; t=2.434 ; p=.018$ ).

Evolución de las creencias a lo largo del tratamiento. El análisis de la evolución de las creencias del grupo de pacientes $\sin$ TP a los tres meses del inicio del tratamiento muestra reducciones significativas del nivel de identificación en siete de las creencias. Por el contrario, en el grupo de pacientes con TP sólo se observan reducciones significativas en la creencia "necesito seguir en tratamiento aunque ya no consuma" (Tabla 1).

La evolución de la media de las creencias del cuestionario a lo largo de los nueve meses de tratamiento se presenta en la Figura 2. Los pacientes sin TP comórbido se identifican progresivamente menos con las creencias a lo largo del tratamiento, si bien es cierto que muestran un ligero repunte en la última evaluación. En el caso de los pacientes con TP la evolución es más irregular pero las diferencias entre las evaluaciones no parecen tan importantes a los nueve meses.

Cuando analizamos la evolución de las creencias "creo que tengo la capacidad de superar mi drogodependencia" indicativa de las expectativas de autoeficacia en función de la presencia o ausencia de TP se aprecian diferencias notables. Mientras que los pacientes sin TP no experimentan apenas variaciones, los pacientes que sí presentan psicopatología en el Eje II mues- tran fluctuaciones acusadas a lo largo del seguimiento. Pese a estas diferencias, las puntuaciones de los dos grupos a los nueve meses son muy similares (Figura 3).

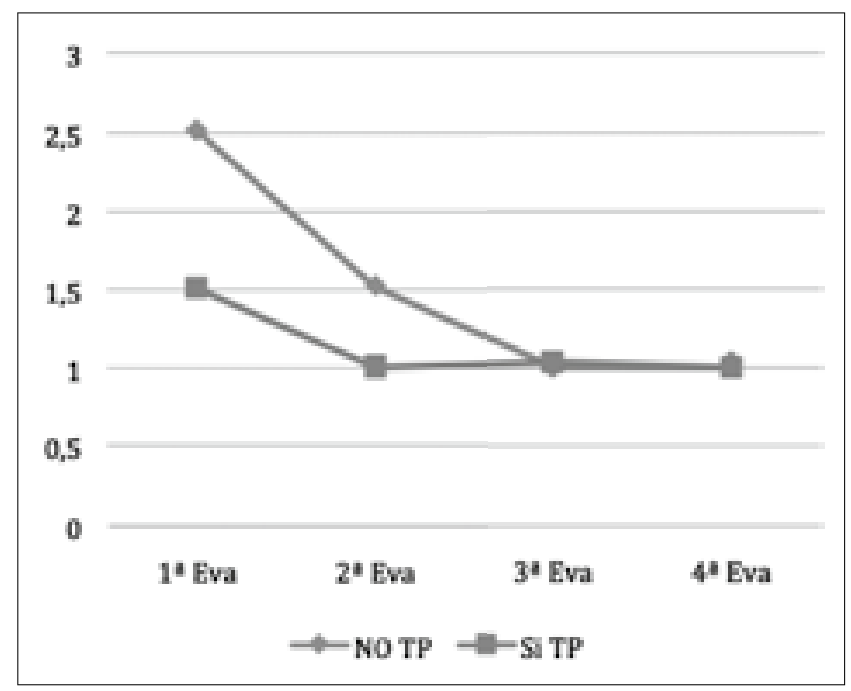

Figura 2. Evolución de las puntuaciones medias del cuestionario.

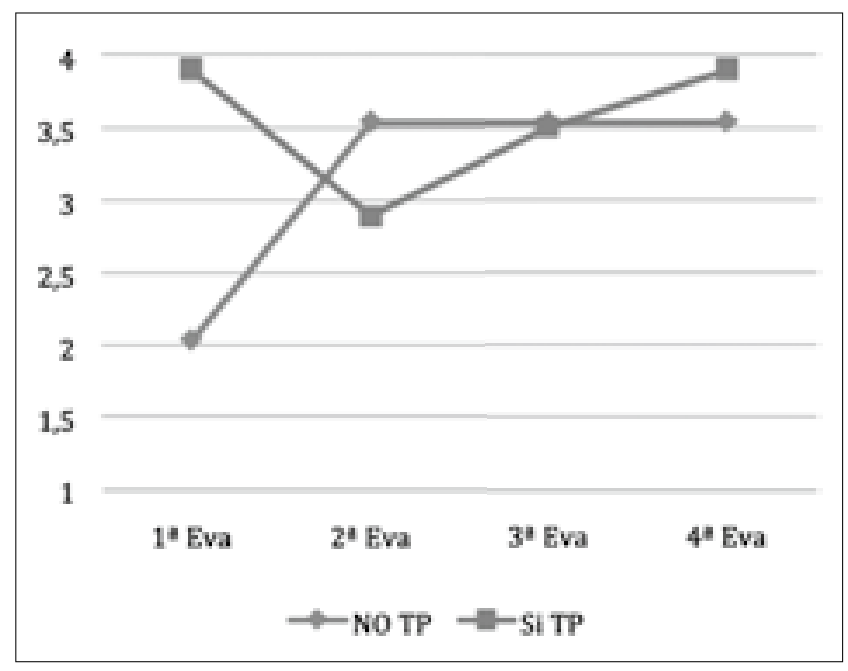

Figura 3. "Creo que tengo la capacidad necesaria para superar mi drogodependencia".

Tabla 1. Diferencias estadísticamente significativas a los tres meses de tratamiento.

\begin{tabular}{|c|c|c|c|c|c|c|}
\hline \multicolumn{7}{|c|}{ Creencias que muestras diferencias estadisticamente significativas a los tres meses del inicio de tratamiento en el grupo de pacientes que no presentan TP. } \\
\hline Creencia & Grupos & Medias & DT & gl & $\mathrm{t}$ & $\mathrm{p}$ \\
\hline \multirow[t]{2}{*}{ Estos problemas me impiden a veces mantenerme abstinente. } & Inicio de tratamiento & 1.25 & 1.712 & 11 & 2.529 & .028 \\
\hline & A los tres meses. & .50 & 1.168 & & & \\
\hline \multirow[t]{2}{*}{ Tengo ganas de consumir cuando me siento bien. } & Inicio de tratamiento & 1.50 & 1.567 & 11 & 2.303 & .042 \\
\hline & A los tres meses. & .58 & .996 & & & \\
\hline \multirow[t]{2}{*}{ Tengo ganas de consumir cuando tengo problemas con alguien. } & Inicio de tratamiento & 2.42 & 1.165 & 11 & 2.200 & .050 \\
\hline & A los tres meses. & 1.42 & 1.564 & & & \\
\hline \multirow[t]{2}{*}{ Creo que la lucha que tengo por estar bien no va a terminar nunca. } & Inicio de tratamiento & .50 & 1.165 & 11 & 2.253 & .046 \\
\hline & A los tres meses. & .33 & 1.564 & & & \\
\hline \multirow[t]{2}{*}{ Me es dificil hacer frente a las ganas de consumir. } & Inicio de tratamiento & 1.17 & 1.030 & 11 & 3.447 & .005 \\
\hline & A los tres meses. & .42 & .515 & & & \\
\hline \multirow[t]{2}{*}{ Mi familia me conoce bien. } & Inicio de tratamiento & 1.17 & .669 & 11 & 2.803 & .017 \\
\hline & A los tres meses. & .42 & .577 & & & \\
\hline \multirow[t]{2}{*}{ Necesito la ayuda de los profesionales. } & Inicio de tratamiento & .33 & .651 & 11 & 2.803 & .056 \\
\hline & A los tres meses. & .08 & .289 & & & \\
\hline \multicolumn{7}{|c|}{ Creencias que muestras diferencias estadisticamente significativas a los tres meses del inicio de tratamiento en el grupo de pacientes que presentan TP. } \\
\hline \multirow[t]{2}{*}{ Necesito seguir en tratamiento aunque ya no consuma. } & Inicio de tratamiento & 2.72 & 2.06 & 17 & 2.486 & .024 \\
\hline & A los tres meses. & 1.589 & 1.392 & & & \\
\hline
\end{tabular}


En relación a la creencia "creo que nunca podré superar mi drogodependencia", mientras que la evolución de las puntuaciones en grupo de personas sin TP es relativamente predecible, la del grupo con TP es más irregular y con cambios poco previsibles (Figura 4).

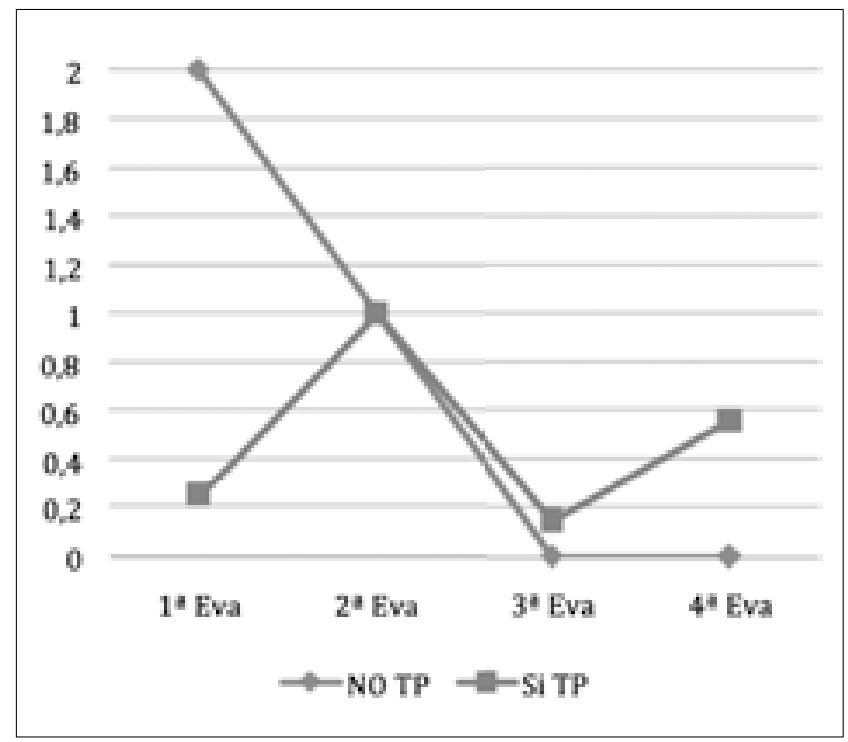

Figura 4. "Creo que nunca podré superar mi drogodependencia".

Mientras que los pacientes sin TP se identifican claramente al inicio del tratamiento con la creencia "algún día consumiré aunque solo sea una vez" "los pacientes con TP apenas muestran identificación con esta creencia. En cambio, el análisis de la evolución muestra un descenso progresivo de las puntuaciones en los pacientes sin TP -que dejan de identificarse con esta creencia a los 6 meses-, tres meses antes que los pacientes con TP, cuyas puntuaciones descienden irregularmente hasta los nueves meses (figuras 5 y 6). En relación a la creencia "algún día consumiré de forma controlada" sucede algo similar aunque en la evolución de los pacientes sin TP se aprecia un nivel inicial inferior y una reducción a los tres meses que se mantiene posteriormente. En el caso de los pacientes con TP el nivel inicial de identificación es mucho mayor, siendo también mucho más lenta la reducción.

Un indicador parcial de la adherencia al tratamiento viene dado por la satisfacción del paciente a lo largo del mismo. La evolución de la creencia "me siento cómodo en el tratamiento" muestra que los pacientes con TP se identifican menos con esta creencia a los nueve meses de tratamiento (figura 7).

\section{Discusión}

De acuerdo con las hipótesis iniciales, el estudio demuestra que es posible medir de manera fiable un conjunto de creencias relacionadas con la adicción y su tratamiento, que el nivel de identificación con las creencias del cuestionario en su conjunto permite predecir el ajuste psicológico global, mientras que el deseo de consumo y los consumos de alcohol durante el tratamiento están vinculadas solo a algunas de las creencias. Además, el curso de dichas creencias durante el tratamiento

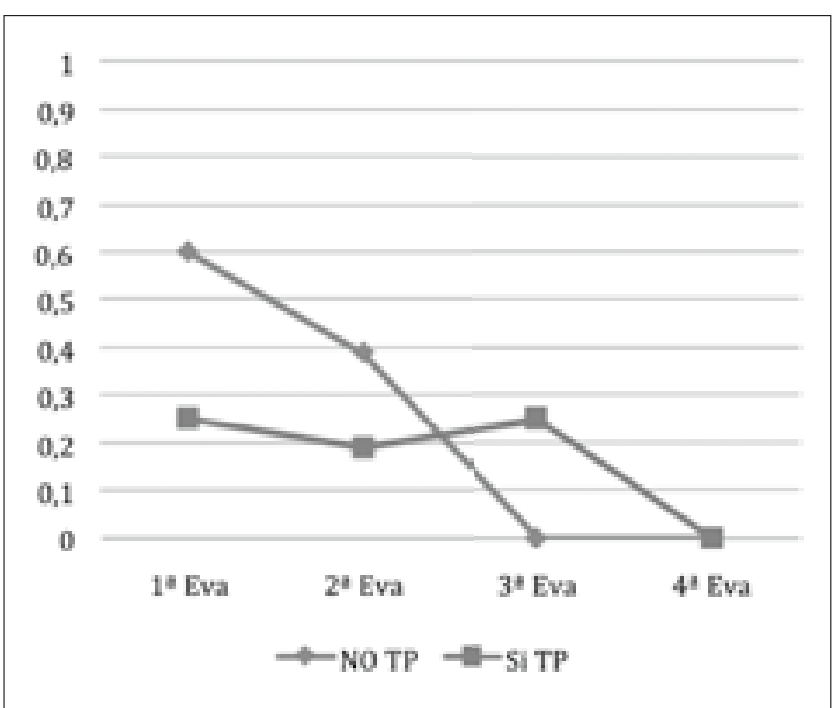

Figura 5. "Algún día consumiré aunque solo sea una vez".

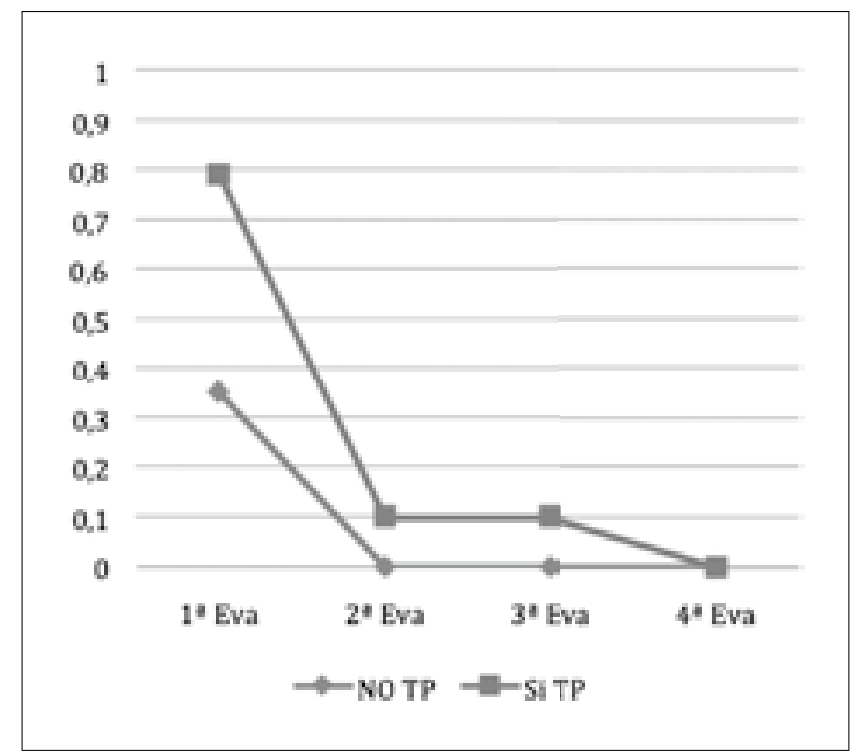

Figura 6. "Algún día consumiré de forma controlada".

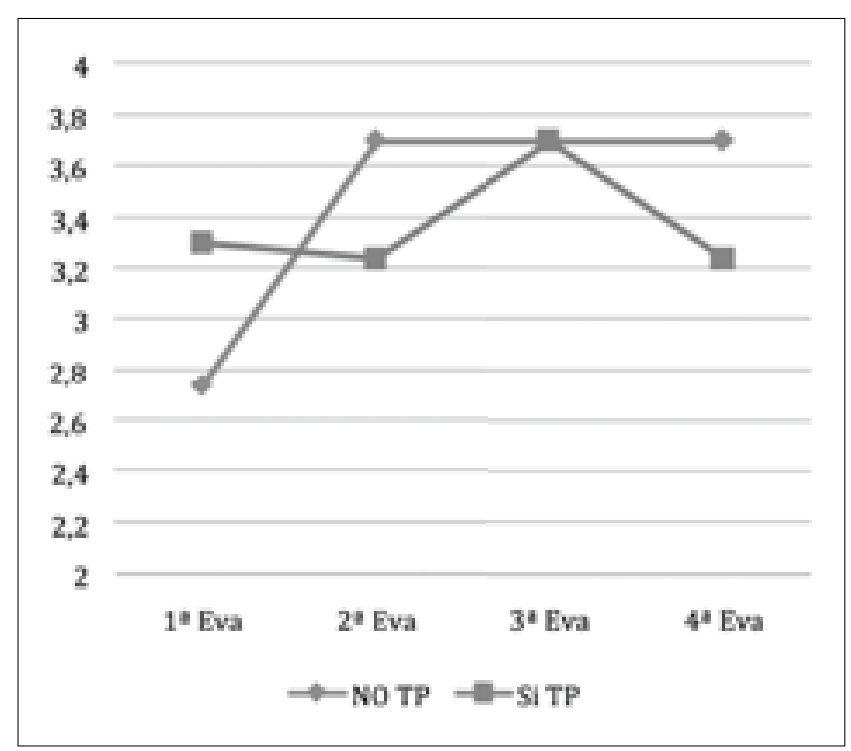

Figura 7. "Me siento cómodo en el tratamiento". 
difiere en función de la presencia de comorbilidad con TP; los pacientes con TP comórbido presentan una evolución mucho más irregular de su identificación con las creencias nucleares relacionadas con la adicción.

Del análisis de la capacidad predictiva de las creencias sobre el craving se extraen aspectos de una enorme importancia para el tratamiento. Puede verse que en relación a la "renuncia", como concepto que aglutina las creencias relacionadas con el propósito del paciente de no volver a consumir drogas, existen tres items que no parecen tener la misma funcionalidad. Mientras que el ítem "consumiré aunque solo sea una vez", es un pensamiento centrado en la experimentación puntual del efecto de la sustancia, las otras dos creencias "consumir de forma controlada" o "tomar un día y no seguir haciéndolo" se refieren al control sobre el consumo y no a la experimentación puntual de la sustancia. Se observan diferencias importantes entre creer que se puede controlar el consumo, como sugieren las dos creencias que no predicen, y la experiencia puntual del efecto del alcohol que no contempla la idea de hacer un consumo controlado, experiencia abiertamente rechazada por los pacientes. Con frecuencia, los pacientes aceptan abiertamente su incapacidad de llevar a cabo un consumo controlado, pero evitan exteriorizan su deseo de volver a experimentar la sustancia aunque solo sea una vez más. Esta relación podría ser un indicador del peso de las creencias permisivas sobre el consumo (Newman y Ratto, 1999).

La correlación entre los ítems relacionados con el concepto de "renuncia" y el deseo de consumo muestra que la renuncia que hacen los pacientes en relación a experimentar nuevamente la sustancia ocupa un lugar destacado entre las variables intervinientes en los proceso de recaídas en el consumo, toda vez que se ha mostrado la asociación de estas creencias con el consumo de sustancias. A su vez, se constata que le índice global de ajuste psicológico influye en el grado con el que los pacientes se identifican con las creencias relativas a la renuncia al consumo, de modo que la creencia sobre la experimentación puntual de la sustancia podría depender en alguna medida de este índice. En los programas de prevención de recaídas deben analizarse junto a las creencias básicas relacionadas con el deseo irrefrenable de consumo de drogas, las asociadas a la recaída en sí misma (Larimer, Palmer y Marlatt, 1999) y el índice de gravedad de ajuste psicológico. A lo largo de los seis primeros meses de tratamiento, se constata el impacto de la creencia "algún dia consumiré aunque solo sea una vez". Todo parece indicar que mientras el paciente se identifique con esta creencia persiste el deseo de consumo.

El hecho de que el ítem "creo que tengo la capacidad necesaria para superar mi drogodependencia" correlacione de forma negativa con los consumos de alcohol pone de manifiesto el peso que tienen las expectativas positivas de autoeficacia en el tratamiento y la abstinencia (Miller, Ross, Emmerson y Todd, 1989). Cuanto más confíe el paciente en su capacidad para afrontar y superar su drogodependencia menos probable es que vuelva a consumir durante los primeros tres meses, que son críticos para el curso del tratamiento. Las diferencias observadas en los ítems "voy a superar mi drogodependencia" y "creo que soy capaz de superar mi drogodependencia" muestra que, aunque ambas creencias se refieren a las expectati- vas de autoeficacia, la creencia que hace referencia explícita a la "capacidad" recoge probablemente más especificamente el componente de autoeficacia. Creencias como "creo que tengo la capacidad para superar mi drogodependencia" y "creo que nunca podré superar mi drogodependencia" predicen el nivel de craving, pero lo hacen en sentido contrario. Una mayor identificación con la primera correlaciona con menor craving, pero sucede al contrario en el caso de la segunda. Esta disociación ilustra la relevancia de los contenidos específicos de las creencias, y cómo las expectativas positivas o negativas de autoeficacia pueden influir en un sentido $u$ otro en el nivel de craving experimentado por los pacientes. Se sabe que las expectativas de autoeficacia pueden modular la intensidad de los síntomas asociados a la adicción (Llorente y Iraurgi, 2008), incluyendo el deseo de consumo. Si la persona no se cree capaz de superar su adicción podría reavivar el deseo, en la medida que considera que su dependencia se prolongará durante largo tiempo. En la experiencia clínica, las expectativas negativas de resultados incrementan el deseo de consumo e incrementan potencialmente, la decisión de ceder a este deseo. Si la persona presenta niveles bajos de expectativa de autoeficacia es más probable que aumente el deseo de consumo, vinculando de este modo las expectativas con la presencia de craving y por extensión con un mayor riesgo de recaída.

En relación a las diferencias de evolución de las creencias entre pacientes con o sin TP comórbidos se han obtenido resultados de gran importancia para el tratamiento. Los pacientes sin TP presentan a los tres meses reducciones adaptativas de su nivel de identificación con siete creencias adictivas, lo que muestra que los pacientes con TP presentan más dificultades para modificar sus creencias nucleares relacionadas con la adicción en los estadios iniciales del tratamiento, tal y como postula el modelo cognitivo de Beck (1995). La trayectoria de evolución de las creencias a lo largo de los nueve meses de seguimiento es cualitativamente distinta en pacientes con y sin comorbilidad: mientras que los pacientes sin TP presentan incrementos o reducciones predecibles, tendentes a la asíntota, los pacientes con TP presentan fluctuaciones abruptas de su nivel de identificación a lo largo del tiempo, demostrando su dificultad para flexibilizar su sistema de creencias de acuerdo con los avances del proceso terapéutico. Esta irregularidad en la evolución incrementa la dificultad del tratamiento de estos drogodependientes y a su vez el riesgo de recaída. Por último, el hecho de que los pacientes con TP presenten niveles más elevados de expectativas negativas de resultados al finalizar el tratamiento plantea la posibilidad de que éstos queden en una situación de mayor riesgo de recaída en comparación al resto de pacientes (Beck, Wright, Newman y Liese, 1999; Verheul, Van den Bosch y Ball, 2007).

\section{Conclusiones}

La evolución de las creencias nucleares relacionadas con la adicción en pacientes drogodependientes con TP, a diferencia de lo que sucede en pacientes sin comorbilidad, muestra que el tratamiento cognitivo es más costoso tal y como se predice en el modelo cognitivo de los TP (Beck, 1995), toda vez que se 
requiere más tiempo para modificar las creencias. No obstante si tenemos en cuenta el peso de estas creencias en los primeros meses de tratamiento - particularmente en pacientes que tienen dificultad para identificar los pensamientos mediante el método socrático -, puede utilizarse el "estilo didáctico" propuesto por Lega, Caballo y Ellis (1997), que consiste en explicar al paciente de manera directa por qué una creencia es irracional. Este estudio plantea la necesidad de focalizar estas técnicas en creencias relacionadas con la "renuncia al consumo" o las "expectativas de autoeficacia y resultado", puesto que están directamente relacionadas con el craving, con la conducta de consumo y con el ajuste psicológico global de los pacientes drogodependientes.

\section{Conflicto de intereses}

Los autores declaran que no tienen conflicto de intereses para este trabajo.

\section{Referencias}

Beck, A. T. y Freeman, A. (1995). Terapia cognitiva de los trastornos de personalidad. Barcelona: Paidos.

Beck, J. (2007). Terapia cognitiva para la superación de retos. Barcelona: Gedisa.

Beck, A. T., Wright, F. D., Newman, C. F. y Liese, B. L. (1999). Terapia cognitiva de las drogodependencias. Barcelona: Paidos.

Chicharro, J., Pedrero, E. y Pérez, M. (2007). Autoeficacia para resistirse al consumo de sustancias como predictora de resultados de tratamiento y su relación con variables de personalidad: estudio de una muestra de adictos con el DICO, el VIP y el MCMI-II. Adicciones, 19, 141-152.

Diclemente, C. C., Fairhurst, S. K. y Piotrowski, N. A. (1995). Self-efficacy, adaptation and adjustment: theory, research and application. New York: Pleunum Press.

DSM-IV-TR (2002). Manual diagnóstico y estadistico de los trastornos mentales, IV. Texto Revisado. Barcelona: Masson.

Golberg, D. P. y Hiller, V. F. (1979). A scaled version of the General Health Questionnaire. Windsor: NFER Publishing Company.

González-Saiz, F. M., Carulla, S., Martínez, J. M., López, A., Ruiz, J. y Guerra, D. (1997). Indicador del tratamiento de la adicción a opiáceos. Cádiz: Servicio de Publicaciones de la Universidad de Cádiz.

Iraurgi, I. y Corcuera, N. (2008). Craving: concepto, medición y terapéutica. Norte de Salud Mental, 32, 9-22.

Larimer, M. E., Palmer, R. S. y Marlatt, G. A. (1999). Relapse prevention: an overview of Marlatt's cognitive-behavioral model. Alcohol Research and Health, 23, 151-160.

Lega, L.I., Caballo, V.E. y Ellis, A. (1997). Teoría y práctica de la terapia racional emotivo - conductual. Madrid: Siglo XXI.
Llorente, J. J. y Iraurgi, E. I. (2008). Tratamiento cognitivo conductual aplicado en la deshabituación de cocaína. Trastornos Adictivos, 10, 252-74.

López, A., y Becoña, E. (2006). El craving en personas dependientes de cocaina. Anales de Psicologia, 22, 205-211.

Long, C. G., Williams, M., Midgley, M. y Hollin, C. R. (2000). Within-program factors as predictors of drinking outcome following cognitivebehavioral treatment. Addictive Behavior, 25, 573-8. doi:10.1016/ S0306-4603(99)00018-0.

López-Ibor, J., Pérez, A. y Rubio, V. (1996). Examen Internacional de los Trastornos de la Personalidad; Módulo DSM-IV. Versión Española. Madrid: Organización Mundial de la Salud.

Martínez-González, J. M., Graña, J. L. y Trujillo, H. M. (2010). La calidad de vida en alcohólicos con trastornos de la personalidad: relación con el ajuste psicológico y el craving. Psicothema, 2, 562-567.

Martínez-González J. M., Graña J. L. y Trujillo, H. M. (2011). Estudio longitudinal sobre calidad de vida, craving y ajuste psicológico en pacientes dependientes del alcohol: variaciones en función de los trastornos de la personalidad. Adicciones, 23, 227-235.

Martínez-González, J. M. y Verdejo, A. (2011). Creencias básicas adictivas y craving. Adicciones, 1, 339-352.

Merikle, E.P. (1999). The subjective experience of craving: an exploratory analysis. Substance Use and Misuse, 34, 1101-1115. doi: 10.3109/10826089909039399

Miller, P. J., Ross, S. M., Emmerson, R. y Todt, E. M. (1989). Self-efficacy in alcoholics: clinical validation of the Situational Confidence Questionnaire. Addictive Behaviors, 14, 217-224. doi:10.1016/03064603(89)90052-X

Newman, C. F. y Ratto, C. L. (1999). Cognitive therapy of substance abuse. In: E. T. Bowd y L. Rugle (Eds), Comparative treatments of substance abuse (pp.96-126). New York: Springer Publishing Company.

Pennay, A., Cameron, J., Reichert, T., Strickland, H., Lee, N. K., Hall, K. y Lubman, D. I. (2011) A systematic review of interventions for co-occurring substance use disorder and borderline personality disorder. Journal of Substance Abuse Treatment, 41, 363-373. doi:10.1016/j.jsat.2011.05.004.

Verheul, R., Van den Bosch, L. y Ball, S. (2007). Abuso de sustancias. En J. M. Oldham, A. E. Skodol, y D. S. Bender, Tratado de los Trastornos de la Personalidad (pp. 465-478). Barcelona: Elsevier Masson.

Tiffany, S. T. (1990). Cognitive model of drug urges and drug abuse behavior: role of automatic and non automatic process. Psychological Review, 97, 147-168. doi: 10.1037/0033-295X.97.2.147 
1. Algún día volveré a consumir un poco de forma controlada.

$\begin{array}{lllll}0 & 1 & 2 & 3 & 4 \\ 0 & 1 & 2 & 3 & 4 \\ 0 & 1 & 2 & 3 & 4 \\ 0 & 1 & 2 & 3 & 4 \\ 0 & 1 & 2 & 3 & 4 \\ 0 & 1 & 2 & 3 & 4 \\ 0 & 1 & 2 & 3 & 4 \\ 0 & 1 & 2 & 3 & 4 \\ 0 & 1 & 2 & 3 & 4 \\ 0 & 1 & 2 & 3 & 4 \\ 0 & 1 & 2 & 3 & 4 \\ 0 & 1 & 2 & 3 & 4 \\ 0 & 1 & 2 & 3 & 4 \\ 0 & 1 & 2 & 3 & 4 \\ 0 & 1 & 2 & 3 & 4 \\ 0 & 1 & 2 & 3 & 4 \\ 0 & 1 & 2 & 3 & 4 \\ 0 & 1 & 2 & 3 & 4 \\ 0 & 1 & 2 & 3 & 4 \\ 0 & 1 & 2 & 3 & 4 \\ 0 & 1 & 2 & 3 & 4 \\ 0 & 1 & 2 & 3 & 4 \\ 0 & 1 & 2 & 3 & 4 \\ 0\end{array}$

2. Tengo ganas de consumir cuando me siento físicamente mal.

3. Cuando se me mete en la cabeza consumir no puedo evitar hacerlo.

4. Creo que nunca podré superar mi drogodependencia.

5. Me siento cómodo en el tratamiento.

6. Tengo problemas emocionales con independencia de mi adicción.

7. [En función de la pregunta anterior] Estos problemas me impiden a veces mantenerme abstinente.

8. Creo que voy a superar mi drogodependencia.

9. Creo que podré tomar un día y no seguir haciéndolo.

10. Tengo ganas de consumir cuando me siento bien.

11. Creo que tengo la capacidad necesaria para superar mi drogodependencia.

12. Creo que aunque deje de consumir no podré hacer una vida normal.

13. Tengo ganas de consumir cuando tengo problemas con alguien.

14. Creo que la lucha que tengo por estar bien no va a terminar nunca.

15. Algún día consumiré aunque solo sea una vez.

16. Mi familia cree que puedo superar mi drogodependencia.

17. Necesito seguir en tratamiento aunque ya no consuma drogas.

18. Me es difícil hacer frente a las ganas de consumir.

19. Tengo ganas de consumir cuando me siento mal.

20. Mi familia me conoce bien.

21. Tengo ganas de consumir cuando dispongo de dinero.

22. Necesito la ayuda de los profesionales.

23. Ahora tengo ganas de consumir. 
